\title{
CORRECTIONS
}

\section{Back on the autistic spectrum}

In Sabina Dosani's Review (BMJ 2012;345:e5470, doi:10.1136/ bmj.e5470) she incorrectly said of the Son-Rise programme for children with autism that its promotion "included a leaflet drop to every member of the UK National Autistic Society [NAS]." However, the charity has told the $B M J$, "The NAS has never and would never hand over the details of our members to a third party without their expressed consent," and Dr Dosani agrees that there is no evidence to support her assertion.

Cite this as: BMJ 2012;345:e5736

๑ BMJ Publishing Group Ltd 2012 